\title{
MedienPädagogik
}

Zeitschrift für Theorie und Praxis der Medienbildung

Themenheft Nr. 30: Medienpädagogik und Erwachsenenbildung. Herausgegeben von Bernhard Schmidt-Hertha und Matthias Rohs.

\section{Erwachsenenbildung und Medienpädagogik: LinkedIn \& Lynda, XING und Google als Bildungsanbieter}

Anke Grotlüschen

\begin{abstract}
Zusammenfassung
Eine der grossen Systematiken der Erwachsenenbildung ist die Unterscheidung in didaktische Handlungsebenen (Mikro-, Meso- und Makroebene), (Schmidt-Lauff 2012), mit Blick auf Internationalisierung und Globalisierung ergänzt um die Mega-Ebene (Egetenmeyer et al. 2017). Diese setzen auf Tietgens didaktischen Handlungsebenen auf. Digitale Möglichkeiten für die Erwachsenenbildung (von Augmented Reality bis Webinar), werden hier in systematisierender Absicht in den didaktischen Handlungsebenen verortet. Dadurch wird erkennbar, dass die Aufgabe der Erwachsenenbildung immer auch die Problematisierung und begründete Entscheidung didaktischer Arrangements enthält. Ein zentraler Aspekt lässt sich jedoch eher schlecht in das klassische Modell einfügen: Die Frage der personalisierten Werbung und der Nutzung bildungsrelevanter Kundendaten wie Lebensläufe und Stellenbörsen. Genau dies machen sich Unternehmen der Digitalwirtschaft (XING, Google, LinkedIn) derzeit zunutze, um Zutritt zum Weiterbildungsmarkt zu erlangen. Entlang dreier Fallanalysen wird versucht - bei begrenztem Zugriff auf Betriebsgeheimnisse eine vorsichtige Einschätzung zu geben, ob es sich hierbei um eine disruptive Entwicklung handelt.
\end{abstract}

Adult education and media education: LinkedIn \& Lynda, XING and Google as training providers

\begin{abstract}
One of the well-known systematic approaches towards adult education is the subdivision into didactical levels of action - micro, meso and macro levels (Schmidt-Lauff 2012). Focusing internationalization and globalization another layer was added, called megalevel (Egetenmeyer et al. 2017). These levels use Tietgen's didactical levels. Digital options for adult education (from augmented reality to webinar) now will be integrated into the layers. Thus it becomes visible that the tasks adult educators have to fulfil, always contain scrutinizing and finding reasonable didactical arrangements. One central aspect cannot be inserted easily into the classical model: The question of personalized advertising and the use of big data like CVs and search engines for vacancies. This is recently being provided by digital enterprises (XING, Google, LinkedIn). They take advantage of their data to enter the training market. This paper presents three case studies to carefully give an estimation whether this is becoming a disruptive development for the training market.
\end{abstract}




\section{Einleitung}

Zum Verhältnis von Medienpädagogik und Erwachsenenbildung ist in jüngerer Zeit wenig Systematisches publiziert worden, eher dominieren Schlaglichter. Die Hessischen Blätter für Volksbildung erlauben unter raumtheoretischer Perspektive einen Blick auf soziale Medien (Iske 2016). Die kürzlich veröffentlichte, durchaus auch an die Praxis adressierte DIE Zeitschrift für Erwachsenenbildung wägt Pro- und Contra-Argumente rund um Digitalisierung ab (Hufer 2017, Rohwerder 2017) und präsentiert instruktionspsychologische Ansätze (Hesse 2017).

Eine Praxistagung des Vereins Weiterbildung Hamburg (2017) präsentiert die Themen der Industrie 4.0 - nicht zuletzt selbstfahrende Autos - sowie Big Data und Learning Analytics als Frage des Marketings. Darüber hinaus werden didaktische Themen angesprochen, u.a. Serious Games, Massive Open Online Courses (MOOC), Webinare und Flipped Classrooms. Der kritische Blick auf den (second level) Digital Divide wird ebenso andiskutiert wie Möglichkeiten der Unterstützung von Menschen mit Behinderungen durch digitalisierte Bildungsangebote. Die Praxis wirft die Frage auf, welche medienpädagogischen Herausforderungen sich für die Erwachsenenbildung stellen. Dabei wird gern konstatiert, man verschlafe disruptive Entwicklungen. Es gilt also zu systematisieren, welche digitalen Entwicklungen in der Erwachsenenbildung neuerdings Einzug halten, welche dieser Entwicklungen disruptiven Charakter haben und ob sie tatsächlich übersehen werden.

Das Konzept der disruptiven Technologien wurde Ende der 1990er Jahre durch den an der Havard Business School lehrenden Clayton M. Christensen entwickelt (Christensen/Matzler 2013). Charakteristisch ist, dass anfangs unterlegene Technologien einen Markt vollständig umwälzen können, dies zumeist unerwartet tun und dadurch etablierte Anbieter existenziell gefährden können. Die These selbst kann durchaus als übertrieben und als aufmerksamkeitsheischend kritisiert werden, allerdings erzeugt sie eine erhöhte Aufmerksamkeit für eine Art von Innovationen, die das Gesamtsystem umwälzt (sog. «game changer»).

Aufgrund der eher unsystematischen Lage der Diskussion über digitale Medien in der Erwachsenenbildung soll zunächst versucht werden, didaktische Szenarien digitalen Lernens in das klassische, etablierte System der erwachsenendidaktischen Theorie einzuordnen. Dies dient der Systematisierung. Darüber hinaus zeigt diese Systematisierung, dass sie die zentrale Frage der Erreichbarkeit von Adressaten (z.B. durch Marketing oder personalisierte Werbung) nicht abbilden kann. Genau deshalb kann es sein, dass hier wichtige Entwicklungen übersehen werden. Das bedeutet noch nicht, dass diese Entwicklungen disruptiven Charakter haben oder dass Disruptivität alle Akteure der Erwachsenen- und Weiterbildung gleichermassen betreffen würde. Nichtsdestotrotz scheint es geboten, am Beispiel jüngerer Aktivitäten zu diskutieren, welche Konsequenzen sich für die Anbieterstruktur ergeben und inwiefern erwachsenendidaktische Theorie sich erneuern muss. Dies dient auch der Erweiterung der medienpädagogischen Professionalisierung in der Erwachsenenbildung (Schmidt-Hertha et al. 2017). 
Digitale Medien auf den didaktischen Handlungsebenen nach Hans Tietgens

Zur Systematisierung bietet sich insofern eine der Kerntheorien der Erwachsenenbildung an, nach der Didaktik in Makro-, Meso- und Mikroebene zu unterteilen ist (Schmidt-Lauff 2012). Dem ging die berühmt gewordene Differenzierung Erwachsenendidaktischer Handlungsebenen durch Hans Tietgens, seinerzeit Leiter der Pädagogischen Arbeitsstelle des deutschen Volkshochschul-Verbandes, voraus (Tietgens 1992, 151). Das Modell entstammt ursprünglich der analogen Welt. Es wurde hinsichtlich eines Megatrends weiterentwickelt, indem der Trias von Mikro-, Meso- und Makrodidaktik die Megadidaktik hinzugefügt wurde, mit der erwachsenenbildungspolitische Aktivitäten im supranationalen Raum bezeichnet werden (Egetenmeyer et al. 2017). Hinsichtlich des Megatrends der Digitalisierung wurde das Modell bisher nicht aktualisiert.

Daher wird es hier als Rahmung verwendet, um Erwachsenenbildung und Medienpädagogik systematisierend aufeinander zu beziehen. Deutlich wird dabei auch, in welcher Weise die Verschiebungen durch digitale Medien die Erweiterung der Systematik didaktischer Handlungsebenen erfordern.

In der durch Tietgens etablierten Theorie didaktischer Handlungsebenen wird die Professionalisierung der Erwachsenenbildung eingefordert, zu ihrer Sicherstellung sei seines Erachtens entsprechende Fortbildung bereitzustellen. Eine solche Fortbildung muss heute auch den Megatrend der Digitalisierung durch entsprechende medienpädagogische Fortbildung abbilden. Derartige Entwicklungen sind in der anwendungsorientierten Forschung durchaus erkennbar (Schmidt-Hertha et al. 2017).

Dennoch ist Tietgens Modell vor einer digital vernetzten Welt entstanden und richtet sich auf eine wesentlich öffentlich finanzierte Erwachsenen- und Weiterbildungslandschaft. Ohne eine Aktualisierung müsste es heute als anachronistisch bewertet werden.

Der Vorteil des Modells liegt allerdings in der Chance, die diversen Bewegungen im Bereich Erwachsenenbildung mit digitalen Medien zu ordnen und entlang dieser Ordnung auf die Gefahr des Übersehens disruptiver Entwicklungen zu prüfen. Mit Tietgens Thesen lässt sich - so sei vorweggenommen - durchaus formulieren, dass die Mehrheit der didaktischen Handlungsebenen eher langsamen, nicht-disruptiven Veränderungen unterliegen. Dem gegenüber ist die Frage der Teilnehmenden- und Kundengewinnung heute unter dem Fokus der Disruptivität neu zu debattieren. Eine entsprechende Analyse wird im zweiten Teil des Beitrags mit Blick auf die Geschäftsmodelle grosser Medienunternehmen vorgenommen. Damit wird auch konstatiert, dass Globalisierung und Digitalisierung in einem erneuerten Modell didaktischer Handlungsebenen bessere Berücksichtigung finden müssen. 


\section{Gesellschaftlicher Bedingungsrahmen (A-Ebene)}

Auf der ersten Handlungsebene lassen sich Rahmungen der digitalen Bildung zuordnen. Das betrifft neue gesetzliche Regelungen (über das Copyright, über Datenschutz), Förderungsrichtlinien und Qualifikationsanforderungen für die Weiterbildung, nicht zuletzt die Frage der Hochschulzulassung und des Marktzutritts für private Hochschulen. Festzuhalten ist hier das lange vor TTIP abgeschlossene «General Agreement on Trade in Services» (Grotlüschen 2002, 2009), das eine grenzüberschreitende Leistungserbringung in der Bildung erlaubt. Damit globalisiert sich das Weiterbildungsangebot, und zwar speziell bei den formalen Langfristformaten - der wissenschaftlichen Weiterbildung mitsamt den Fernstudien und ihrem neuen Format der Massive Open Online Courses (MOOC). Besondere Marktvorteile haben englischsprachige Angebote und Zertifizierungen international renommierter Institute und (Ivy-League-) Universitäten.

\section{Institutionelle Voraussetzungen (B-Ebene)}

Die Zielsetzungen von Bildungsinstitutionen sind unterschiedlich. Die öffentlich geförderten Bildungsträger verpflichten sich dem Gemeinwohl und der Teilnehmenden-Orientierung, die privatwirtschaftlich agierenden Institute streben nach institutionellem Selbsterhalt und «Shareholder Value». Institutionen haben demzufolge Präferenzen für Veranstaltungsformate - von fünftägiger Bildungsfreistellung gewerkschaftsnaher Bildungsträger bis zur Vortragsreihe wissenschaftsnaher Bildungsträger - die in unterschiedlicher Weise durch Digitalisierung berührt sind. Die Vortragsreihe kann durch Skype-Vorträge und Webinare durchaus profitieren, die Bildungsfreistellung hingegen lebt von der Zusammenkunft und der Distanz zum Alltag. Dennoch sind Serious Games, Plan- und Rollenspielformate und Augmented Reality auch in diesem Setting sinnvoll zu etablieren.

Aufgrund geringerer Teilnahmezuweisungen und variabler Kurseinstiege haben sich die Anbieter im SGB III-Bereich zunehmend der Digitalisierung zugewandt. Diverse Träger bieten vor Ort Computer-Selbstlernzentren mit vielfältiger Lernsoftware an, bei denen ein laufender Einstieg möglich ist (u.a. Sprach- und Buchhaltungskurse). Bundesweite Träger bieten zudem Blended-Learning-Formate für Umschulungen an, die über Bildungsgutscheine in Anspruch genommen werden können, für die aber lokal keine hinreichenden Gruppenstärken erreicht werden.

\section{Aufgabenbereichsstruktur im Fachbereich (C-Ebene)}

Didaktik auf dieser Ebene enthält Annahmen über Teilnehmende und strukturiert das Angebot entsprechend. Dazu gehört das Verhältnis von Routine-Veranstaltungen und Innovationen, auch in Fragen des (digital unterstützten) Formats sowie der (durch 
Digitalisierung induzierten) Auswahl der Inhalte. Nicht zuletzt ist hier die Zielgruppenarbeit angesiedelt, die im Hinblick auf digitale Medien die Frage nach erster und zweiter digitaler Spaltung aufwirft. Neue Themenfelder richten sich unter anderem auf digitale Grundbildung und digitales Schreiben (Thillosen 2008; Lea 2005).

\section{Angebotsgestaltung (D-Ebene)}

Hier sind laut Tietgens Eigenheiten der Sachstruktur und Lernstruktur zu verhandeln. Als Sachstruktur unter dem Vorzeichen der Digitalisierung ist heute die Content-Entwicklung einschliesslich Open Educational Resources zu verstehen. Diese beinhaltet dann auch zentrale didaktische Entscheidungen. Digitale Medien verändern die Möglichkeiten des exemplarischen Lernens (Negt 1969), der didaktischen Reduktion und der didaktischen Anreicherung (Siebert 2009). Die mit digitalen Medien lernenden Teilnehmenden können sodann systemseitig mitverfolgt und durch entsprechend didaktisches Eingreifen unterstützt werden (Learning Analytics). Prüfungsbestimmungen sind als Teil des gesellschaftlichen Bedingungsrahmens festgelegt, auf dieser Ebene jedoch reflektiert umzusetzen. Das schliesst Varianten des E-Assessments ein. Zur Angebotsgestaltung gehören jedoch auch der Ankündigungstext und der Distributionskanal.

\section{Veranstaltungsverlauf (E-Ebene)}

In der Veranstaltung selbst steht laut Tietgens die Dramaturgie der Lehr- und Lernprozess-Gestaltung im Vordergrund. Hier erlaubt die Digitalisierung eine Reihe von Ansätzen, die lerntheoretisch informierten Qualitätsansprüchen durchaus entgegenkommen. Erkundungen, Projekte, Recherchen, Produktionen und Ausstellungen sind durch den Einsatz mobiler Geräte und persönlicher Lernumgebungen bzw. Portfolios eher erleichtert worden. Mobile Learning-Angebote können vor Ort historische Informationen bereitstellen (Die Berliner Mauer) und durch Augmented Reality-Formate lassen sich Kameraabbild (z.B. einer Bergkette, einer Pflanze) und bildungsrelevante Information zusammenbringen (z.B. Benennung der Berge und Pflanzen, Entwicklung von Gletschern, Vulkanaktivität und Biotopen). Die häufig begleitend entwickelten Audioguides von Museen und Ausstellungen lassen sich inzwischen auf Smartphones laden und durch weitere mobile Informationen anreichern, verlieren aber dadurch nicht die didaktische Problematik, zwischen Belehrung und Belanglosigkeit zu einer angemessenen Vermittlung von Gegenstand und Lernenden zu finden, wie qualitative Studien zeigen (Eggert 2009; Nolda 2007).

Nicht zuletzt sind YouTube-Tutorials für eine Reihe von Bildungsveranstaltungen seriös einsetzbar, u.a. das berufspädagogische Projekt draufhaber.tv (Wolf/Rummler 2011). Dasselbe gilt für Lernspiele, beispielweise «Winterfest»-Spiel des Deutschen 
Volkshochschul-Verbands, das zur Alphabetisierung und Grundbildung dient. Das UNHCR hat wiederum ein Spiel über Fluchtrealitäten aufgelegt (Last Exit Flucht, http://www.lastexitflucht.org/againstallodds/).

\section{Erweiterungsbedarf des Modells didaktischer Handlungsebenen}

Zunächst bietet diese in den 1990er Jahren entstandene Ebenen-Systematik eine noch immer tragfähige Struktur der erwachsenenpädagogischen Handlungen bei klassischen Bildungsträgern, vor allem der Volkshochschule. Sie erlaubt die Einordnung digitaler Entwicklungen auf der Ebenenstruktur und unterstützt somit die Systematisierung des Verhältnisses von Erwachsenenbildung und digitalen Medien. Jedoch kann sie kaum abbilden, in welchem Ausmass inzwischen projekt- und marktförmig gearbeitet wird. Weiterhin hat die Frage der Teilnehmenden-Ansprache inzwischen vollständig neue Formen angenommen. Diese professionellen Handlungsprobleme sind im Verhältnis von Medienpädagogik und Erwachsenenbildung bisher meines Wissens nicht systematisch diskutiert worden. Die Frage, in welcher Weise digitale Medien zu neuen Marketingstrategien führen, ist mit dem klassischen Ansatz der didaktischen Handlungsebenen von Hans Tietgens heute nicht mehr sinnvoll abzubilden. Das zeigt sich auch daran, wie unbemerkt sich neue Akteure in der Weiterbildungslandschaft platzieren können. Es liess sich keine einzige Publikation in den deutsch- oder englischsprachigen Fachdatenbanken finden, die dieses Phänomen bereits aufgegriffen oder gar kritisch gewürdigt hätte (über das Lehren und Lernen mit sozialen Medien und über die Präsenz von Weiterbildungsanbietern in sozialen Medien gibt es durchaus Beiträge, z.B. Angelis et al. 2013; Wankel et al. 2010). Während also die bisher diskutierte Entwicklung eher als kontinuierlich zu bezeichnen wäre, muss hinsichtlich des Bildungsmarketings und der Distributionsformen noch einmal gefragt werden, ob es sich hier um disruptive Entwicklungen handelt und ob sie möglicherweise in der Erwachsenenbildungspraxis und -forschung bisher übersehen werden.

\section{Disruptive Entwicklungen in der Weiterbildungsbranche?}

Disruptive Prozesse sind Prozesse, die ein bestehendes Gefüge nicht langsam, sondern auf einen Schlag verändern. Diese Prozesse können lang etablierte, stabile und grosse Akteure in relativ kurzer Zeit vernichten. Die Digitalisierung der Fotografie hat beispielsweise die Laborentwicklung auf ein Minimum zurückgefahren und die Fabrikation analoger Filme fast vollständig ausgesetzt. Marktführer wie Kodak, Spartenanbieter wie Ilford und Massenanbieter wie Foto Dose mit all seinen Filialen sind kurzerhand aus dem Angebot verschwunden und das Fotoalbum ist dem Fotobuch gewichen. Ähnliche Entwicklungen zeigen sich im Film- und Musikmarkt - Vinyl und 
CD sind dem Streamingdienst gewichen. Auch Erwachsenen- und Weiterbildung kann in Streamingform mit Abonnement und sozialer Community ausgebracht werden. So werden zum Beispiel seit Mitte 2017 Videovorlesungen der «ZEIT Akademie» mithilfe der Amazon Prime-Infrastruktur angeboten.

Während die durch öffentliche Mittel teilfinanzierten Bildungsträger im Wesentlichen vor sukzessiven, stetigen, durchaus existenzbedrohenden, aber insgesamt eher langsamen Kürzungen der Förderkulisse stehen, sehen sich die privatwirtschaftlich agierenden Institute offenbar mit neuer Konkurrenz und substanziell neuen Geschäftsmodellen konfrontiert.

Verknüpfbare Datenbestände über Weiterbildung, Veranstaltungsangebote, Stellenangebote und Lebensläufe

Das Besondere an den hier zu diskutierenden neuen Geschäftsmodellen liegt in der digitalen Verfügbarkeit der Produkte, in globalen Vertriebsmärkten und im Streaming anstelle des Erwerbs von Produkten. Das allein genügt jedoch nicht, um im Weiterbildungsmarkt disruptive Entwicklungen voranzubringen. Eine disruptive Dynamik entsteht erst, wenn eine sehr genaue Bedarfsanalyse möglich ist - und diese gelingt auf Basis von Social Media und monopolisierten Märkten. Hier treffen drei Arten von Datenbeständen aufeinander.

1. Es liegen in grossem Umfang Lebenslaufdaten online vor, oft gekoppelt an berufliche Interessengebiete und berufliche Position (Facebook, LinkedIn, XING).

2. Es liegen in grossem Umfang Stellenausschreibungen online vor, mit Angabe der gesuchten Studienabschlüsse, Berufserfahrungsjahre und Kompetenzen (Stepstone, Monster, LinkedIn, XING)

3. Es liegen in grossem Umfang Kursangebote online vor, in diversen Sprachen und mit Ankündigungstexten, die die Qualität professioneller Werbetexte aufweisen (LinkedIn Learning \& Lynda.com, Google Trainingszentren)

Diese drei Arten von Datenbeständen erlauben den genannten Unternehmen, sehr gezielte Weiterbildungswerbung zu platzieren und ihre Angebote auszubringen.

\section{Quasi-monopolistische Maklerfunktion in nahezu vollständigen Märkten}

Damit greifen XING und LinkedIn nicht etwa die klassischen Bildungsträger an, die Konkurrenz bezieht sich eher auf die bisherigen privatwirtschaftlichen Anbieter. Möglicherweise haben die Provider Sozialer Netzwerke dabei auch lediglich Interesse an einer Maklerfunktion, so dass Dritte hier ihre Kursangebote vermarkten können. Diese Maklerfunktion gibt es derzeit in zunehmend oligopol- oder monopolähnlicher Form für verschiedenste Dienste: 
- Stellenbörse (z.B. Stepstone, Monster, indeed)

- Wohnungsbörse (z.B. Immobilienscout24, Immonet, Immowelt)

- Partnerbörse (z.B. Parship und Tinder)

- Einkaufsbörse (z.B. Amazon)

- Flohmarkt (z.B. Ebay)

- Reiseinformationen (z.B. Tripadvisor, HRS)

- Flugbuchungen (z.B. Google Flights)

- Ferienzimmervermietung (z.B. Airbnb)

- Musik- und Videostreaming (z.B. Netflix, Spotify)

Charakteristisch ist für viele dieser Börsen der makelnde Charakter, es wird also nur vermittelt, der eigentliche Geschäftsprozess verläuft nicht einmal durch die Systeme des Maklers - teilweise wird bereits für die Buchung auf Drittanbieter verwiesen (z.B. bei Tripadvisor oder Google Flights). Bildungsmakler würden dann ähnlich agieren wie monopolistische Shopping- oder Flohmarktmakler, wie Wohnungssuchmaschinen und Partnerbörsen: Sie stellen das Produkt nicht her, sondern verknüpfen Produkt und Kundschaft mit Hilfe ihres Datenbestands - und sie diktieren die Regeln, nehmen für jeden Vertragsabschluss eine Gebühr (Amazon, Ebay, AirBnB, Uber) bzw. lassen sich den Zugang zur Kundschaft oder zum Produkt durch Abonnements oder Monatsraten bezahlen (Partnerbörsen, Musik- und Videostreamingdienste). Google hingegen nimmt an, dass die Digitalisierung massenhaft Entlassungen und ergo hohe Umschulungsbedarfe zur Folge haben wird und stellt neue Berufsbilder vor, die in den hauseigenen Trainingszentren erlernt werden können (gratis).

\section{Fehlende Marktdaten in der Erwachsenen- und Weiterbildung}

Disruptiv sind Entwicklungen nicht zuletzt dann, wenn sie konventionelle Lösungen, Prozesse und Produkte in bemerkenswerter Geschwindigkeit irrelevant werden lassen. Hier wäre insofern zu fragen, welche Bereiche der Erwachsenen- und Weiterbildung durch die neuen Marketingstrategien der genannten drei Unternehmen der Digitalwirtschaft überhaupt berührt werden.

Dazu lohnt ein Blick auf die so genannten «Lünendonk-Listen», die über Jahre hinweg die führenden (d.h. genauer: umsatzstärksten) Anbieter der beruflichen Weiterbildung berichtet haben. Das Ergebnis zeigt, dass diese Listen seit 2007 nicht mehr erstellt werden. Lünendonk hat sich dem (offenbar lukrativeren) Personaldienstleistungsmarkt zugewandt. Diese Entwicklung ist m.E. nicht der Digitalisierung, sondern der neuen Gesetzgebung im Arbeitsmarkt geschuldet, durch die SGB-III-geförderte Umschulung stark reduziert und die Personaldienstleistung stark ausgebaut wurde.

Die früheren Lünendonk-Listen zeigten jedoch relativ deutlich, dass unter den umsatzstärksten Anbietern der beruflichen Weiterbildung immer auch grosse 
Hard- und Softwarehersteller waren (SAP, Hewlett-Packard, IBM). Diese haben in aller Regel nicht im öffentlich geförderten Erwachsenen- und Weiterbildungsmarkt agiert, sondern profitorientiert und begleitend zur eigenen Produktentwicklung einen «Schulungsmarkt» entwickelt. Im Freizeitbereich war Ähnliches bei den Kameraherstellern zu beobachten, die seit dem Ausbringen neuer, komplexer Digitalkameras zunehmend Tutorials bereitstellen, mit denen sowohl die Kamerabedienung als auch die kreative Fotografie thematisiert werden. Eine Einschätzung, ob aktuelle Entwicklungen auf Basis nutzbarer Datensätze (Lebensläufe, Stellenangebote) nunmehr disruptiven Charakter für die öffentlich geförderte und privatwirtschaftlich agierende Weiterbildung haben werden, lässt sich derzeit (u.a. wegen fehlender Marktdaten wie ehemals den Lündendonk-Listen) nicht auf quantitativer Basis treffen. Daher folgt nunmehr eine Beschreibung von Geschäftsmodellen. Sie soll als eine weitere Annäherung an das Problem der Disruptivität gelten.

Weiterbildungsrelevante Geschäftsmodelle grosser Medienunternehmen (Google, XING, LinkedIn)

Drei Geschäftsmodelle sollen nachfolgend vorgestellt werden, sofern sie von aussen einsichtig sind. Grundsätzlich fussen zwei dieser Modelle auf der Verknüpfung von Stellenmarkt, Lebenslaufdaten und Kursangeboten (XING und LinkedIn). Die zwei Netzwerke (LinkedIn und XING) fungieren als Makler, die Bildungsangebote werden von Dritten ausgebracht. Demgegenüber nutzt Google seine interne Kenntnis digitaler Marketingstrategien und skizziert auf dieser Basis neue Berufsbilder. Diese Berufsbilder werden dem Szenario der Industrie 4.0 mit weithin veränderten Berufstätigkeiten entgegengesetzt. Dabei kooperiert Google mit klassischen Bildungsanbietern.

\section{XING: Das Event-Markt-Modell einer Plattform für Seminare, Stellenanzeigen und Wei- terbildung}

Das deutschsprachige Berufstätigen-Netzwerk XING lässt derzeit keine eigenen Aktivitäten als Bildungsanbieter erkennen. Allerdings ist XING wie auch LinkedIn auf gezielte Stellenangebote spezialisiert.

Weiterhin bietet XING personalisierte Werbeanzeigen, die dem eigenen Berufsprofil entsprechen. Selbstverständlich hat die persönliche Suchhistorie einen Einfluss auf die Werbe- und Stellenangebote. Unter den Werbeangeboten befinden sich im zum Beispiel Weiterbildungsangebote, etwa von der «digital Marketing School» oder der «WBS Akademie». Hinzu kommen in der Werbeleiste so genannte «Events», also messeähnliche Veranstaltungen, die im weiteren Sinne auch der (informellen) Weiterbildung und Vernetzung zuzurechnen sind. Auch Kurzzeitseminare werden 
gelegentlich als «Event» in XING angelegt. Sie sind nach Stadt sortierbar und mit Kalenderfunktion sowie Google Maps verlinkt. Dies wird durchaus zum Marketing für kostenpflichtige Kursangebote genutzt, z.B. durch die Digital Marketing School (siehe Abbildung).

\section{XING ${ }^{X}$}

n > Eventmarkt > Hamburg > Seminare, Workshops und Vorttäge in Hamburg > Certified Digital Marketing Professional ( 5 Tage, Hamburg)

\section{Certified Digital Marketing Professional}

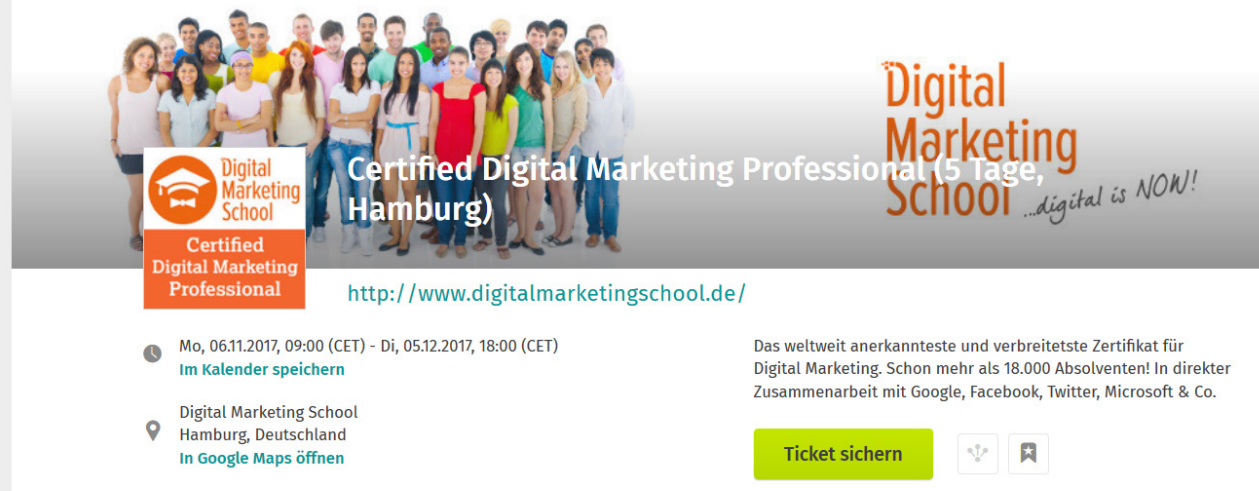

aiz Ticket sichern

Abb. 1.: Screenshot XING, Kursangebot Digital Marketing School (Quelle: Screenshot Anke Grotlüschen).

Die Eventdatenbank auf XING enthält gegenwärtig über 19.000 Einträge, die von Vortragsreihen grosser Zeitungen über alternative Heilmethoden bis zu Design und dem Übergang ins papierlose Büro reichen. Eine Testsuche unter dem Stichwort «Train the Trainer» ergab 200 Veranstaltungsangebote, darunter die klassischen Angebote der Industrie- und Handelskammern. 


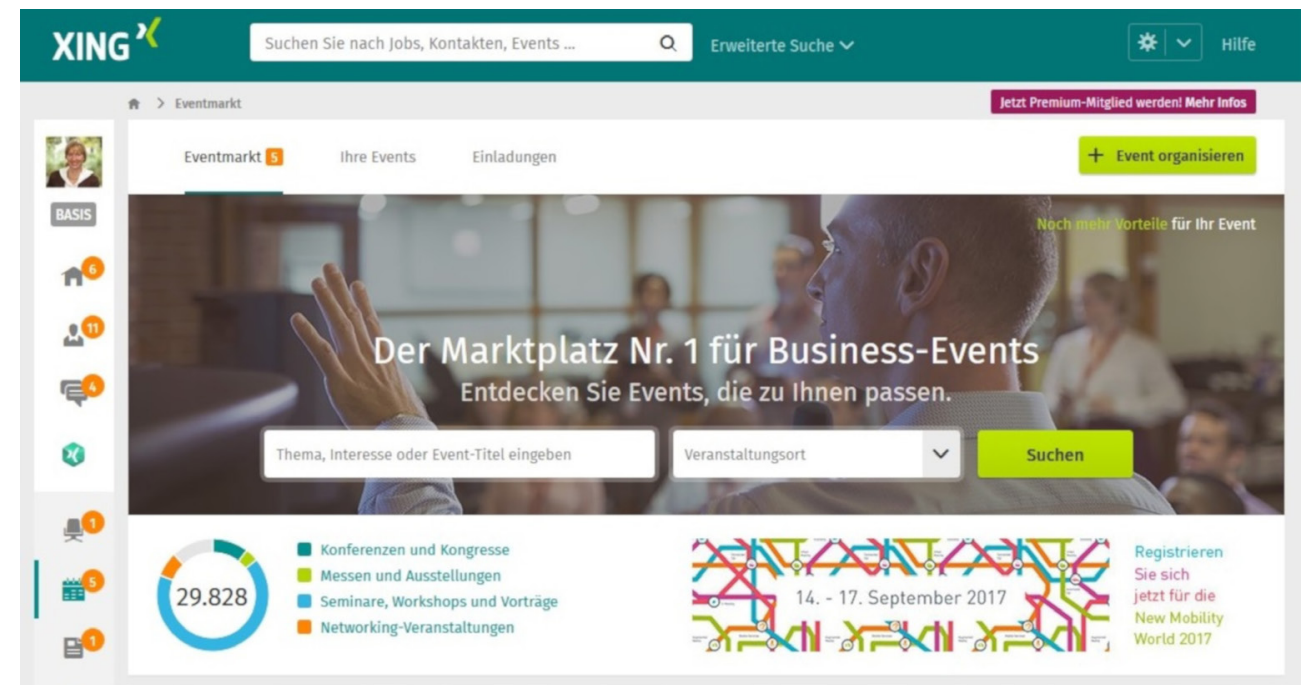

Abb. 2.: Screenshot XING, Eventdatenbank (Quelle: Screenshot Anke Grotlüschen).

Das Event-Tool enthält selbstverständlich alle Vernetzungsangebote, die ein soziales Medium zu bieten hat, darunter die Verknüpfung mit bisherigen Nutzerinnen und Nutzern, deren Bewertung des Angebots und des Anbieters - so wie jede Stellensuche auch Informationen darüber erbringt, ob man bei dem angezeigten Arbeitgeber Kontakte ersten oder zweiten Grades hat, ob man sich bei ihnen über den Arbeitgeber erkundigen möchte und ob man seinen eigenen Arbeitgeber anonym bewerten möchte.

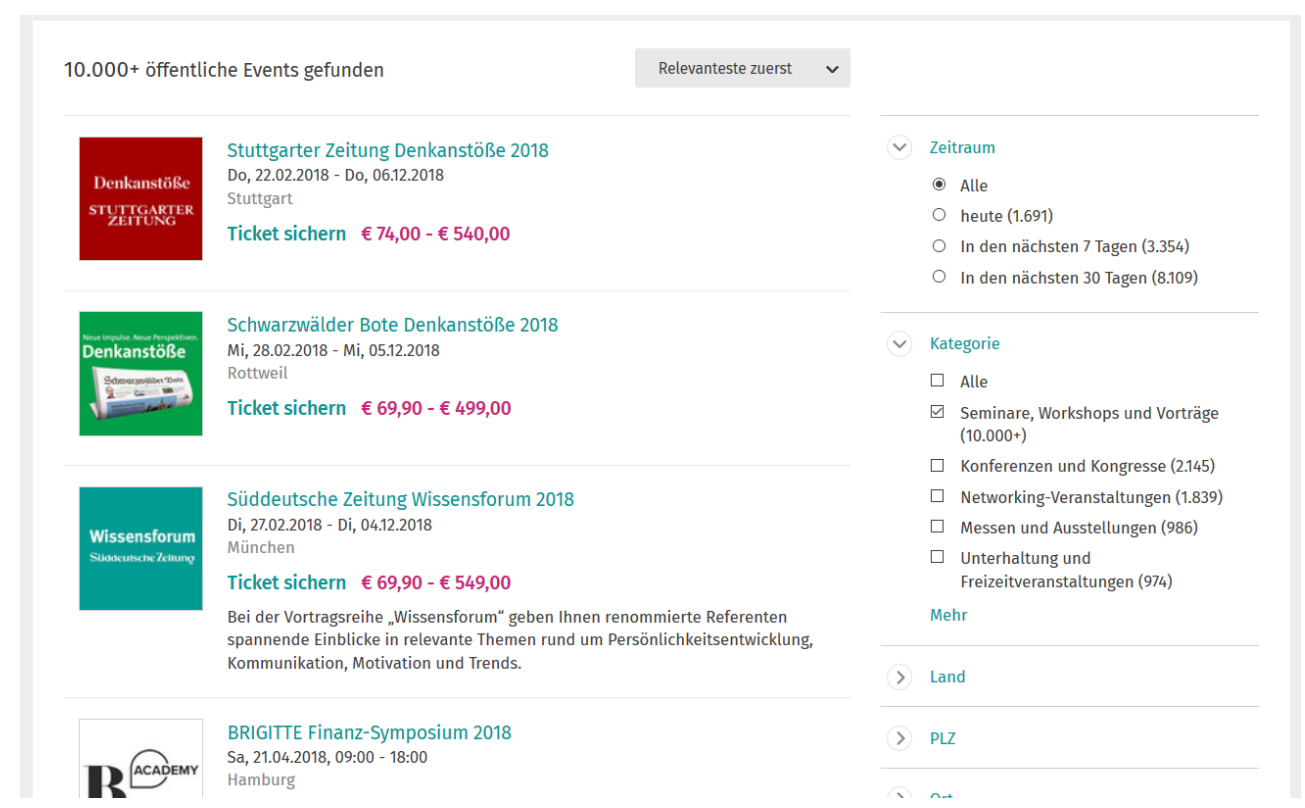

Abb. 3.: Screenshot XING, öffentliche Events (Quelle: Screenshot Anke Grotlüschen). 
Insgesamt sind hier, im deutschsprachigen Weiterbildungsmarkt, auch einige grosse Träger der öffentlich (ko-)finanzierten Weiterbildung aufzufinden, die eine solche Marketingstrategie bereits nutzen. XING etabliert sich dabei insofern als EventMarktplatz, nicht als Bildungsanbieter. Es ist also nicht von einer disruptiven Entwicklung auszugehen, sondern von einer weiteren, auf soziale Medien ausgerichteten Marketingstrategie, mit der die bisherigen Strategien (Zeitungswerbung im Stellenanzeigenteil, Werbung im öffentlichen Raum) ergänzt werden. Interessant ist die Überschneidung von Seminar, Event und Messe, die in einer solchen Struktur eher in den Veranstaltungsteilen der Fachmagazine der Personalwirtschaft auftritt (Manager Seminare, Training Aktuell, Zeitschrift Personalwirtschaft, Brand Eins). Diese Magazine richten sich eher an Berufstätige aus dem Human Resources Management, nicht aber an den Endkunden. Umgekehrt wird inzwischen in jedem Bewerbungstraining die digitale Stellensuche geübt. Ergo ist damit zu rechnen, dass zunehmend an beruflicher Weiterbildung interessierte Personen auf XING und LinkedIn anzutreffen sind. Dadurch ist die Reichweite dieser Werbestrategie nicht grösser, aber präziser.

Insbesondere kann XING den bisherigen Lebenslauf der suchenden Person einbeziehen, um passende Stellen- und Weiterbildungsangebote anzuzeigen. Das führt dazu, dass die obige Recherche fast ausschliesslich Angebote für Personen mit Berufserfahrung erbringt - mit Schwerpunkt im Bereich der öffentlichen Verwaltung sowie in der (digital affinen) Weiterbildung. Berufseinsteigerinnen und Berufseinsteiger berichten hingegen davon, dass ihnen in erheblichem Ausmass Coaching-Angebote zur Berufsorientierung angezeigt werden. Die eingegebenen Daten des Lebenslaufs (Jahrgang des Schulabschlusses, des Studienbeginns, Standort der Hochschule) lassen sich relativ schnell zu einer Zielgruppe für derartige Angebote aggregieren.

Insgesamt ist bei XING eine Maklerstrategie im Eventbereich zuzüglich personalisierter Werbung zu Weiterbildungen und Stellenangeboten zu verzeichnen. Bemerkenswert ist die enge Verknüpfung von Lebenslauf, Stellenanzeigen und Kompetenzen. Je mehr Daten durch Nutzerinnen und Nutzer eingegeben werden, umso wertvoller wird das Netzwerk für die zahlenden Anzeigekunden. Je mehr Angebote es verzeichnet, umso eher sehen sich die Nutzerinnen und Nutzer genötigt, ihre Stellen- und Weiterbildungssuche, ihre Messe- und Jubiläumsveranstaltungsbesuche über XING zu betreiben und die jeweils erworbenen Credits (Kompetenzen) auch im eigenen Lebenslauf zu vermerken und durch Dritte bestätigen zu lassen. Auf Dauer entsteht ein Monopol für den deutschen Sprachraum.

\section{LinkedIn Learning: Das Flatrate-Modell für Grosskunden}

Die Social Media Plattform LinkedIn ist ein Vernetzungstool für Berufstätige, das vor allem international verwendet wird. Es können Gruppen eingerichtet, Nachrichten erstellt und weitergegeben werden und es bestehen Möglichkeiten, zu einem Bereich 
der Stellenangebote zu wechseln. LinkedIn wurde im Jahr 2016 von Microsoft übernommen. Seinerseits nutzt LinkedIn Lehr- und Lernangebote des Anbieters Lynda. com. Es handelt sich insofern um eine Marktbewegung, aus der in aller Vorsicht auf ein gewisses kommerzielles Interesse an Berufsnetzwerken, Stellenbörsen und Weiterbildungsbörsen geschlossen werden kann.

Doch bereits ohne den Wechsel zum Angebot von Lynda.com finden sich auf den LinkedIn-Seiten Menübereiche, in denen spezielle Lernangebote vorgehalten werden. Dieses als LinkedIn Learning titulierte Angebot ist nur scheinbar gratis: Selbst für die Testnutzung sind eine Reihe persönlicher Daten anzugeben, die dann zur telefonischen Kontaktaufnahme durch das Unternehmen führen. Zur Vervollständigung der Fallanalyse wurden die Daten der Autorin angegeben, um Testzuzgang zum Unterrichtsmaterial zu erhalten. Es erfolgte ein deutschsprachiger Anruf aus Dublin. Erst danach wurde ein schriftliches Angebot unterbreitet. Es erlaubt eine «Kostprobe», nämlich den «Organizational Learning and Development» von Britt Andreatta. Daher wird diese Kostprobe hier kurz skizziert.

Der Kurs «Organizational Learning and Development» ist wie folgt spezifiziert: «In order to attract and retain talent, organizations need to provide their employees with the opportunity to learn and grow in their careers. As a result, learning and development programs (L\&D) have become a critical talent management tool, helping leaders, managers, and recruiters build their talent pipeline. In this course, expert and consultant Britt Andreatta shares how to build an L\&D program for your organization to attract and retain talent as well as develop the skills that keep your organization competitive. She shares how to adapt your program over time. She helps you assess your organization's level of maturity and then walks you through the best practices of designing a learning program that maps to your organization's maturity level. She covers adult learning theory and methods, outlines the basics of instructional design, and explains the different kind of learning programs available to you.» (Andreatta, Britt. 2016. "Organizational Learning and Development (LinkedIn Learning)». Video und Kurs: 1 Std. 32 Min.(4. November). https://www.linkedin.com/learning/organizational-learning-and-development.) 


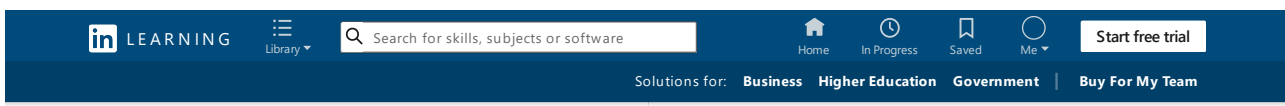

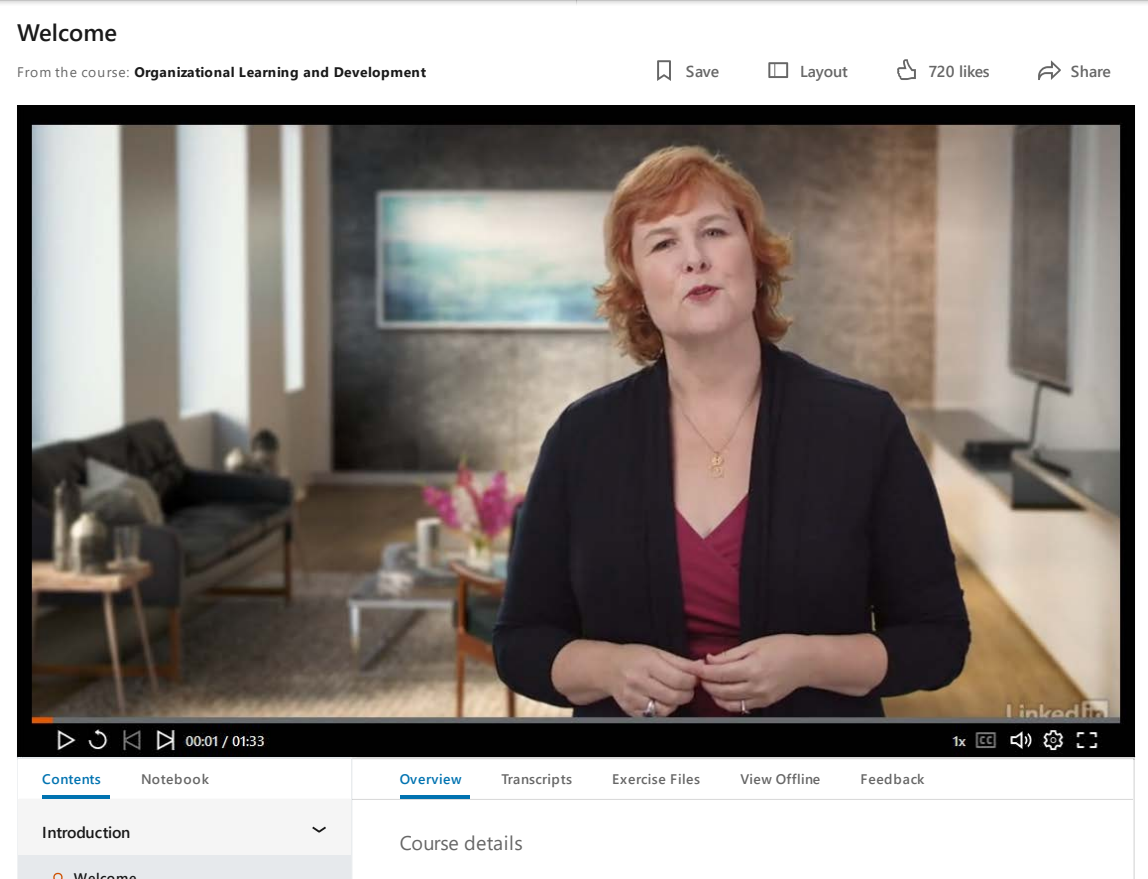

Abb.4.: Screenshot LinkedIn Learning, Kurs: "Organizational Learning and Development», Trainerin: Britt Andreatta (Quelle: Screenshot Anke Grotlüschen).

Trainerin: Britt Andreatta. «I help people and organizations rise to their potential» (Angabe mit Bild und Link zum LinkedIn-Profil der Trainerin)

Kursinhalt:

«Introduction/ welcome (1. Min. 33 Sek.)

1. Organizational Learning and Development Overview

- The stages of organizational development

- Importance of learning to organisational development

- Understand your organization's L\&D stage

- Being a strategic partner to the business

- The ROI of talent development

- Creating a culture of learning

2. Best Practices for Effective Learning

- Adult learning theory

- Neuroscience of learning

- Creating real behavior change

- Adult learning methods

- Assessing your audience

- Instructional design: Story arc + insight 
- Instructional design: Format + delivery

- The critical nature of practice

3. Elements of Organizational L\&D

- Choosing between bespoke or off-the-shelf-solutions

- Learning program systems

- Learning program processes

- Curating content

- Evaluation methods

- Conclusion

- Next steps»

Ankündigungstext aus LinkedIn Learning bzw. darin eingebettet Lynda.com, Zugang nur mit Abonnement (https://www.linkedin.com/learning/organizational-learning-and-development).

Dem hier gezeigten «Kursinhaltsverzeichnis» folgt der Unterpunkt «ähnliche Kurse», nämlich The Neuroscience of Learning und Instructional Design: Adult Learners, mit etwa einer Stunde Dauer.

Nun wäre das Ausbringen videographierter Vorträge mitsamt Reflexionsübungen, Arbeitsblättern und Musterlösungen nicht besonders originell, wären da nicht einige Details, die im hier unternommenen Selbstversuch aufgefallen sind. Erstens ist die Kurs-Video-Qualität ausgesprochen professionell im Vergleich zu YoutubeVideo-Tutorials oder videographierten Vorlesungen. Zweitens sind die etwas unter fünf Minuten liegenden Abschnitte in sich geschlossen, sie werden drittens aber in direkter Folge abgespielt, so dass eine Verlockung entsteht, noch etwas weiter dabei zu bleiben. Wie zu erwarten ist, folgt nach einer Vorschau dann der kostenpflichtige Bereich, der nur mit einer Flatrate zugänglich ist.

Unterhalb der Kursbeschreibung ist erkennbar, wie viele Personen diesen Kurs angesehen haben (hier: 20.811 Personen), in welchen Bereichen sie tätig sind (hier: Unternehmensausbilder, Recruiter, Spezialist-Personalwesen und Universitätsprofessor). Diese sind unter anderem beschäftigt bei IBM, der US Army, Tata Consultancy Services, Deloitte und PwC. Der Kurs deckt laut LinkedIn folgende Kenntnisse ab: Training \& Development, Employee Learning \& Development, People Development, Learning \& Development, Staff Development und Talent Management. LinkedIn hält die Möglichkeit vor, diese Kompetenzen zum eigenen Profil hinzuzufügen. Sie können durch Dritte aus dem eigenen Netzwerk «bestätigt» werden. So entstehen Profile, die für Recruiter aussagestark sind und für das Individuum Vorteile auf dem Arbeitsmarkt erbringen - sofern sich alle Beteiligten dieser Logik unterwerfen wollen. 

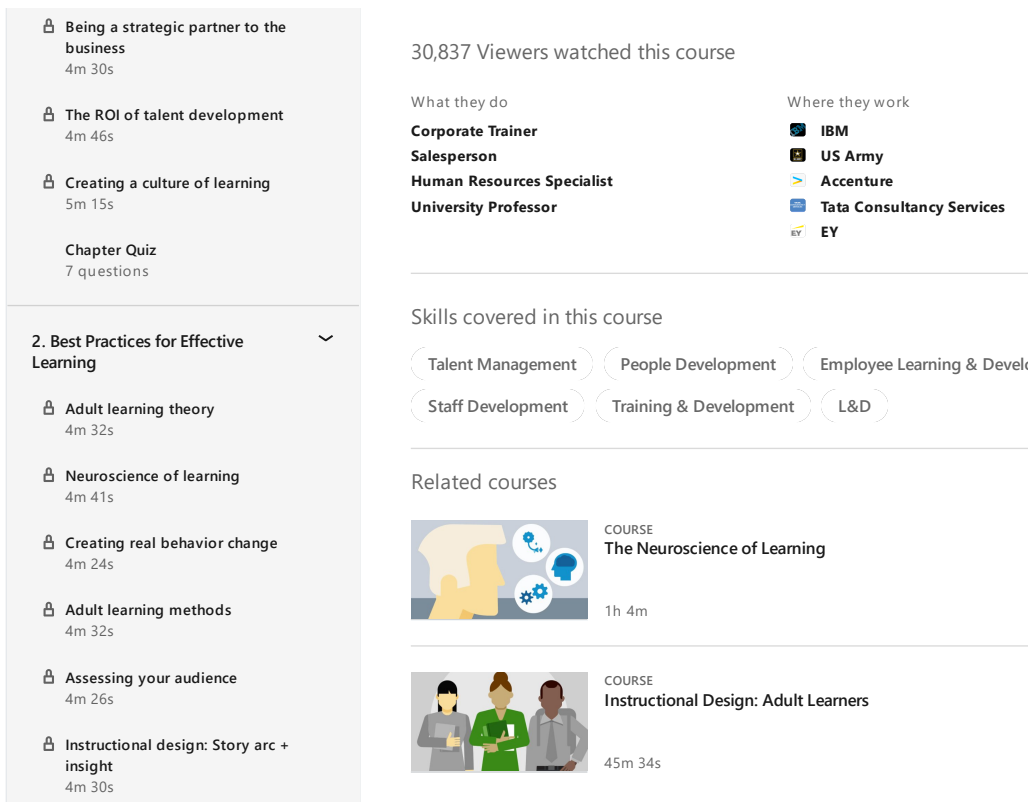

Skills covered in this course

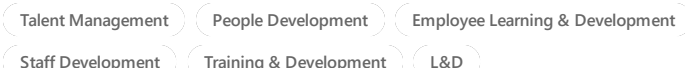

Staff Development Training \& Development L\&D

Related courses
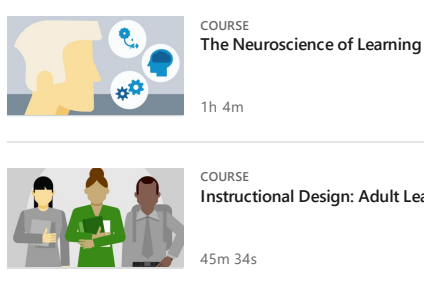

COURSE

COURSE
Instructional Design: Adult Learners

$45 \mathrm{~m} 34 \mathrm{~s}$

Abb.5.: Screenshot Linkedln Learning, kursrelevante und personenbezogene Informationen unterhalb der Kursbeschreibung (Quelle: Screenshot Anke Grotlüschen).

Das deutschsprachige LinkedIn-Angebotsspektrum der Kurse wird in einem Gesamtdatenblatt im schriftlichen Angebot mit unterbreitet. Es richtet sich nicht allein auf individuelle Lernende. Vielmehr bieten LinkedIn und Lynda Firmenlösungen an, die sie den Human Resources Development-Abteilungen bzw. der Organisations- und Personalentwicklung anbieten (entsprechend ist das Gratisangebot auch für die Personalentwicklung zugeschnitten). Das bestehende Kursangebot richtet sich weitgehend auf Software und - interessanterweise - auf kreative Fotografie (eventuell ist das Kursangebot durch Aufkäufe oder Kooperationen entstanden, das könnte die Sparten erklären). Im Gesamtangebot ist der Bereich der Führungskräfteentwicklung, der sonst in der betrieblichen Weiterbildung zu den Standardangeboten gehört, relativ selten vertreten. Möglicherweise werden diese direkt in englischer Sprache ausgebracht.

Das von LinkedIn nach der telefonischen Kontaktaufnahme unterbreitete Kursangebot enthält 2.000 deutschsprachige sowie 6.000 englischsprachige Kurse, zusätzliche Kurse liegen auf Spanisch, Französisch oder Japanisch vor. Die Kurse können in Form von Playlists geordnet werden, Funktionen sind Lesezeichen, Untertitel, unterschiedliche Abspielgeschwindigkeit, Notizenfunktion und Offline-Modus. Weiterhin kann die Playlist «kuratiert» werden, zudem sind «Kursempfehlung nach Nutzungsverhalten und Nutzerprofil mit spezifischen Interessensgebieten erstellbar» (Angebotstext August 2017). Es werden Teilnahmenachweise erstellt «mit der Möglichkeit diese und diese in sozialen Netzwerk zu posten» (ebd.). Innerhalb der 
Videokurse werden Arbeitsblätter aufgerufen, diese liegen als Dateien, auch als Projektdateien und Coding-Testumgebungen vor. Die Videotexte liegen transkribiert vor und Übungsdateien sind ebenfalls im Hauptmenü abrufbar.

Für die Personalentwicklung ist weiterhin von Bedeutung, dass ganze Betriebe für die Kursmodule freigeschaltet werden können. Die Nutzung durch Betriebsangehörige kann anschliessend berichtet werden (individuelles und für Arbeitsgruppen aggregiertes Reporting). Weiterhin können betriebsspezifische eigene Kurse entwickelt werden. Die Streaming-Lizenz pro Person und Jahr liegt bei rund 300 Euro, ein Gratismonat ist möglich.
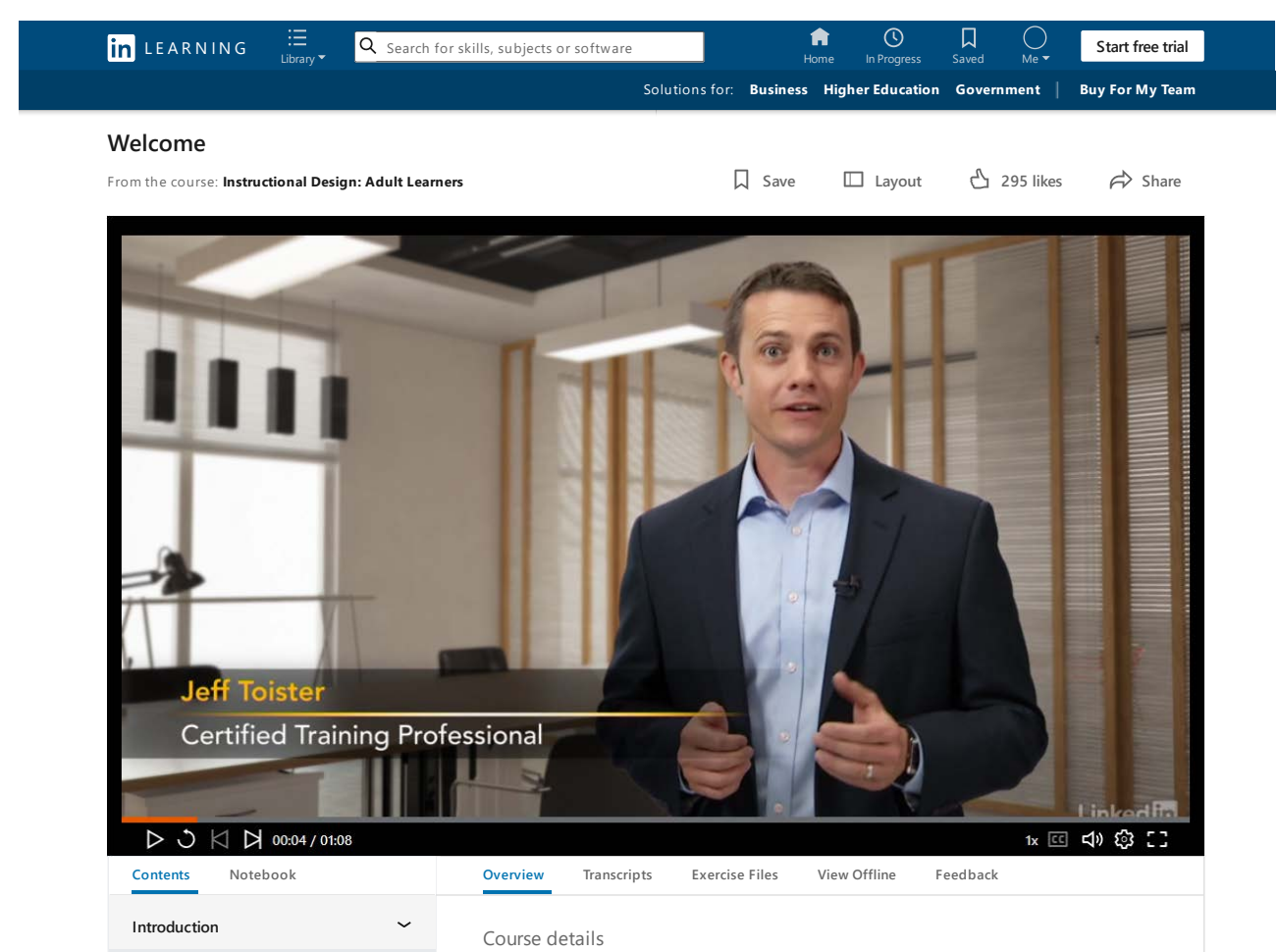

Abb. 6.: Screenshot LinkedIn Learning, Kurs: «Instructional design: Adult Learners» Trainer: Jeff Toist (Quelle: Screenshot Anke Grotlüschen).

Etwas überraschend scheint bei LinkedIn und Lynda die Preispolitik mit einer Flatrate von 300 Euro im Jahr, die noch oberhalb der Flatrates der Musik- oder Serienund Fernsehdienste liegt. Ungewöhnlich erscheint auch die Distribution von GratisModulen, die erst nach einem Telefonkontakt ermöglicht wird. Beides deutet darauf hin, dass das LinkedIn sein Angebot als Business-to-Business-Geschäft versteht, bei dem die Flatrates für Kurspakete an Personalabteilungen grösserer Betriebe verkauft werden sollen. Dem widerspricht zwar das Angebot an Fotographiekursen, dennoch ist die Einstiegsstruktur insgesamt an Personalabteilungen ausgerichtet. 
Die Frage, ob dieses Geschäftsmodell disruptiven Charakter birgt, hängt insofern eng mit der Einkaufspolitik der Betriebe zusammen. Für gesetzlich vorgeschriebene Weiterbildungen (Sicherheitsschulungen, Gender-Mainstreaming-Vorschriften) ist eine digitale Ausbringung von Kursmaterialien vermutlich nicht mehr ungewöhnlich. Im Führungskräftesegment wird Weiterbildung jedoch auch als Incentive und als Vernetzungsinstrument eingesetzt. Diese Elemente kann LinkedIn Learning kaum abdecken, hier ist insofern nicht von disruptiven Entwicklungen auszugehen.

\section{Google Zukunftswerkstatt: Das Content-Modell in Trainingszentren vor Ort}

Google hat im Sommer 2017 eine bundesweite Werbekampagne gestartet, um aktuell aufgesetzte Weiterbildungsangebote bekannt zu machen. Dazu gehören vier mehrseitige Heftbeilagen in der Süddeutschen Zeitung sowie Plakatwerbung und entsprechende Werbung in den Timelines der sozialen Medien wie Facebook. Startpunkt ist München:

«Die Zukunftswerkstatt soll von München aus die digitale Bildung in ganz Deutschland voranbringen und besteht aus zwei übergeordneten Programmen, wie ein Google-Sprecher erläutert. Die Zukunftswerkstatt richtet sich zum einen an Arbeitgeber, Arbeitnehmer und Auszubildende in Unternehmen, Vereinen und Universitäten. Sie können sich online oder in Workshops in der realen Welt mit Themen wie Online-Marketing, Kommunikation, Web-Analyse oder Design-Thinking beschäftigen.» (SZ vom 17.7.2017)

Mit dieser Positionierung ist vor allem die berufliche Aus- und Weiterbildung angesprochen.

«Zudem können Klassen künftig auf eine virtuelle Klassenfahrt gehen, den sogenannten Google Expeditions, die gemeinsam mit der Stiftung Lesen entstanden sind. Die Schüler bekommen Virtual-Reality-Brillen, der Lehrer steuert das System über ein Tablet. So können die Jugendlichen zum Beispiel zur Hamburger Elbphilharmonie reisen oder an Orte, zu denen sie im wirklichen Leben gar nicht fahren könnten, wie zum Mond oder ins Innere des menschlichen Körpers.» (SZ vom 17.7.2017)

Die obige Aussage verweist jedoch auch auf die - seit langem bekannten - Begehrlichkeiten grosser Konzerne wie Google und Microsoft, verstärkt zum schulischen Bildungsbereich Zugang zu erlangen. Weniger üblich waren solche Aktivitäten jedoch im Weiterbildungsmarkt. Das mag daran liegen, dass der Weiterbildungsmarkt bisher nicht als sehr lukrativ angesehen wurde. In seiner Beilage zur Süddeutschen Zeitung vom Mai 2017 skizziert Google jedoch eine Reihe neuer Berufsbilder und konstatiert, dass im Zuge der Industrie 4.0 klassische Aufgaben wegfallen und neue Berufstätigkeiten entstehen werden. Aus dieser Lücke wird dann ein Weiterbildungsangebot 
begründet. Die angesprochenen Berufsbilder lauten (SZ Beilage 2017, 11-12; 35-36, Beschreibungen gekürzt, AG):

- SEM Specialist/-in (Search Engine Marketing - Spezialist/-in, stellt Auffindbarkeit von Webseiten sicher)

- Social Media Manager/-in (steht im Dialog mit Nutzerinnen und Nutzer und Kundschaft)

- Content Manager/-in (erzeugt Inhalte in Form von Fotos, Texten und Videos)

- 3-D-Artist/-in (gestaltet dreidimensionale Objekte und Räume, auch für Virtual Reality Anwendungen)

- Mobile Developer/-in (entwickeln und testen Apps für Mobiltelefone)

- UX Designer/-in (stellt die geeignete User Experience bzw. das Nutzernden-Erlebnis her, indem Surfverhalten analysiert wird und einfache Bedienbarkeit erzeugt wird)

- SCRUM Master/-in (stellt verteiltes, selbstorganisiertes Programmieren sicher)

- Cloud-Architekt/-in (betreibt und begleitet die Umstellung der lokalen IT mitsamt Speicher, Anwendungen und Diensten ins Netz)

- Lösungsarchitekt/-in (Entwickelt Lösungen beim Kunden, passt Programme an Geschäftsabläufe an)

- Category Manager/-in (Warengruppenmanagement im Einzelhandel und onlineShops)

- Datenwissenschaftler/-in (werten Daten einer Firma aus und bereiten sie für Abteilungen auf

Die so beschriebenen Berufe werden als neu und attraktiv, gegebenenfalls auch als sicher dargestellt. Die Anspracheform ist erkennbar jugendlich. In der Heftmitte der SZ-Beilage befinden sich sodann vier Seiten zur Google Zukunftswerkstatt, die kostenlose Kurse erläutern und zur Anmeldung auffordern. Angeboten werden

- Basiswissen über die online-Darstellung, Soziale Medien

- Marketing über Google, Auswertung durch Google Analytics, gemeinsames Arbeiten mit Google-Produkten

- Tools für den (Schul-)Unterricht (Google Expeditions, open Roberta)

- Unterstützung von Gemeinnützigkeit durch Google.org, Google Ad Grants

Drei Städte eröffnen eigene Trainingszentren (München, Hamburg, Berlin), weitere Städte wurden durch eine Art Roadshow im Sommer und Herbst 2017 bespielt. Neben den realen Kursangeboten findet sich online - ebenfalls gratis - ein Angebot an Lektionen, die als E-Learning-Module bearbeitet werden können. Bemerkenswert ist die Strategie, die Angebote sämtlich gratis auszubringen. Damit erzeugt Google neue Nutzerdaten, macht seine Produkte bekannt und verdrängt die Konkurrenz vom Weiterbildungsmarkt. Besonders auffallend ist die Konzentration auf Deutschland mit 
dieser Strategie. Partner der Trainingsangebote sind mehrere Hochschulen und Bildungsträger (IHK München und Oberbayern, Stiftung Lesen). Auch professorale Unterstützung konnte Google gewinnen, wie der Berichterstattung zur Eröffnung des Münchner Trainingszentrums zu entnehmen ist (Prof. Dr. Gesche Joost, Professorin für Design an der Universität der Künste Berlin, durch die Bundesregierung ernannte Internetbotschafterin, http://www.faz.net/aktuell/wirtschaft/digitale-bildung-google-eroeffnet-zukunftswerkstatt-in-muenchen-15110213.html).

Die Datenschutzinformationen, die bei der Anmelde-Aufforderung vor der Nutzung eines online-Tutorials abrufbar sind, zeigen sehr eindeutig, dass sämtliche Daten von Google und Partnern verknüpft und zu Marketingzwecken von Google verwendet oder den Google-Kunden zur Verfügung gestellt werden.

Insgesamt ist das Google-Angebot sehr an klassische Weiterbildungsanbieter gekoppelt. Google tritt nicht als Flatrate-Anbieter wie LinkedIn oder als Eventmakler wie XING auf, sondern generiert Weiterbildungsinhalte auf Basis einer Behauptung, die zukünftig relevanten Berufsbilder zu kennen. Mit seinem Content-Gratisangebot werden Nutzerdaten generiert und Marktanteile gewonnen.

Konsequenz für die medienpädagogische Professionalisierung des Personals in der Erwachsenenbildung

Tietgens kommt der Verdienst zu, für die Erwachsenenbildung einen Begriff von Didaktik entwickelt zu haben, der weit über die mikrodidaktische Unterrichtsgestaltung hinausgeht. Dadurch werden auch mega-, makro-, mesodidaktische Handlungen als pädagogische Handlungen begreifbar. Medienpädagogische Kompetenz ist in der Erwachsenenbildung somit nicht ausschliesslich auf mikrodidaktische Prozesse gerichtet, sondern beinhaltet auch die Lehrgangsplanung, Programm(bereichs-)planung und die Gestaltung gesellschaftlicher Rahmenbedingungen. So kommt auch die Frage der Geschäftsmodelle und Marktbewegungen in den Blick. Die Positionierung von Weiterbildungsangeboten ist damit keine betriebswirtschaftliche, sondern eine pädagogische Handlung.

Deutet man didaktisches Handeln im Verhältnis zur Medienpädagogik, würde sich somit die Frage stellen, welche medienpädagogischen Aufgaben hinsichtlich des Geschäftsgebarens der Digitalwirtschaft für das Personal der Erwachsenenbildung entstehen.

Geschäftsmodelle und Marketingstrategien unterliegen somit ebenso der Digitalisierung wie die weiter oben den didaktischen Handlungsebenen zugeordneten digitalen Elemente. Bisherige medienpädagogische Weiterbildung (z.B. EBmooc, Aschemann 2017) nimmt auf den Markteintritt der Digitalwirtschaft in die Weiterbildungsbranche noch keinen Bezug. Das in der Entwicklung befindliche Modell zur Medienkompetenz von Lehrenden in der Weiterbildung (Schmidt-Hertha et al. 2017) 
wird diese Elemente möglicherweise aufnehmen. Dabei wäre weiter zu beobachten, wie sich das Event-Modell (XING), das Flatrate-Modell (LinkedIn Learning) und das Content-Modell (Google) längerfristig entwickeln.

Insgesamt ist bisher keine aktualisierte Marktübersicht, keine einschlägige medienpädagogische Weiterbildung und auch keine entsprechende Medienkritik im Fachdiskurs zu verzeichnen, die über den Markteintritt der Digitalwirtschaft berichtet. Es handelt sich um eine Verschiebung in der Weiterbildungslandschaft, die wesentlich durch personalisiertes Marketing, ergo die Nutzung von Datenbeständen gekennzeichnet ist.

Ob diese Entwicklung disruptiv ist, kann derzeit kaum entschieden werden. Allerdings hat sie das Potenzial dazu, weil die Verknüpfung von Lebenslauf, Stellenmarkt und Weiterbildungsmodulen derart verkaufsträchtig ist, dass sie kaum zu verhindern sein wird - obwohl dabei grosso modo sensible Daten preisgegeben werden. Es wird in der medienpädagogischen Fortbildung insofern auch darauf einzugehen sein, wie diese Entwicklung kritisch begleitet und differenziert genutzt werden kann. «Digitaler Selbstmord» (also die vollständige Abstinenz von Berufstätigen bzw. von Bildungsträgern aus digitalen Sozialen Netzwerken) wird sicher keine Lösung sein.

\section{Literatur}

Angelis, Kylene D., Nadia Catenazzi, Melanie Graham, Michael Klebl, Viktorija Mazeikiene, Giedrẻ Valūnaitė Oleškevičienè, Kirsty Palfreyman, Kirsty Reid, Jolita Šliogerienès, Jarno Tolonen, und Jeffrey van Zaalen. 2013. ISTUS report. Institutional strategies for the uptake of social media in adult education. http://ebooks.mruni.eu/product/institutional-strategies-for-uptake-social-media-in-adult-education-istus-report.

Aschemann, Birgit. 2017. "Die digitale Bereitschaft der Erwachenenbildung. Der EBmooc für ErwachsenenbildnerInnen». Magazin erwachsenenbildung.at 11 (30). http://nbn-resolving. de/urn:nbn:de:0111-pedocs-128928.

Christensen, Clayton M., und Kurt Matzler. 2013. The innovator's dilemma: Warum etablierte Unternehmen den Wettbewerb um bahnbrechende Innovationen verlieren. 1., korrigierter Nachdr. München: Vahlen.

Egetenmeyer, Regina, Sabine Schmidt-Lauff, und Vanna Boffo. 2017. «Internationalization and Professionalization in Adult Education: An Introduction». In Adult Learning and Education in International Contexts: Future Challenges for Its Professionalization: Comparative Perspectives from the 2016 Wurzburg Winter School, hrsg. v. Regina Egetenmeyer, Sabine Schmidt-Lauff, and Vanna Boffo, 9-24. Studies in pedagogy, andragogy, and gerontagogy Vol. 69. Frankfurt am Main: Peter Lang. https://flore.unifi.it/retrieve/handle/2158/1079360/222956/PL_FR_267875_Egetenmeyer_ak_P2.pdf.

Eggert, Barbara. 2009. «Der Audioguide als Element der Lernkultur im Museum - Untersuchungen zum intendierten Hörer». In Transkulturelle Perspektiven auf Kulturen des Lernens, hrsg. v. Wiltrud Gieseke, Steffi Robak, und Ming-Lieh Wu, 215-42. Bielefeld: transcript Verlag. 
Grotlüschen, Anke. 2002. «E-Learning im Weltmarkt der Dienstleistungen - GATS.» Hessische Blätter für Volksbildung 52: 210-22.

Grotlüschen, Anke. 2009. «Konsequenzen der Globalisierung - Fernstudium und Bildungsdienstleistungen.» Hessische Blätter für Volksbildung (2), 131-39. Globalisierung und Erwachsenenbildung. Herausgegeben von: Hessischer Volkshochschulverband e.V. Bielefeld. doi:10.3278/HBV0902W.

Iske, Stefan. 2016. «Erwachsenenbildung und der soziale Raum informeller Bildung im Internet.» Hessische Blätter für Volksbildung (1), 82-91. Lernräume. Herausgegeben von: Hessischer Volkshochschulverband e.V. Bielefeld. doi:10.3278/HBV1601W.

Lea, Mary R. 2005. «CCommunities of Practice» in Higher Education: Useful Heuristic or Educational Model?» In Beyond Communities of Practice: Language, Power and Social Context, hrsg. v. David Barton und Karin Tusting, 180-97. Cambridge: Cambridge University Press.

Negt, Oskar. 1969. Soziologische Phantasie und exemplarisches Lernen: Zur Theorie der Arbeiterbildung. Frankfurt am Main: Europäische Verlagsanstalt.

Nolda, Sigrid. 2007. «Green Disaster Three Times: Vermittlungsformen kulturellen Wissens in Perspektivverschränkung.» In Weiterbildung am Beginn des 21. Jahrhunderts: Festschrift für Wiltrud Gieseke; eine Publikation des Erwachsenenpädagogischen Instituts Berlin e.V, hrsg. v. Ulrike Heuer, Ruth Siebers, and Wiltrud Gieseke, 61-73. Münster: Waxmann.

Schmidt-Hertha, Bernhard, Matthias Rohs, Rott, Karin Julia, und Ricarda Bolten. 2017. «Fit für die digitale (Lern-)Welt? Medienpädagogische Kompetenzanforderungen an Erwachsenenbildner/-innen». DIE Zeitschrift für Erwachsenenbildung (3): 35-37. doi:10.3278/DIE1703W035.

Schmidt-Lauff, Sabine. 2012. "Professionelle Handlungsfelder und -ebenen in der Erwachsenenbildung.» Enzyklopädie Erziehungswissenschaft Online, 1-22. doi:10.3262/ EEO16090010.

Siebert, Horst. 2009. Didaktisches Handeln in der Erwachsenenbildung: Didaktik aus konstruktivistischer Sicht. 6., überarb. Aufl. Grundlagen der Weiterbildung. Augsburg: Ziel.

Thillosen, Anne M. 2008. Schreiben im Netz: Neue literale Praktiken im Kontext Hochschule. Münster: Waxmann. https://www.waxmann.com/?elD=texte\&pdf=2061Volltext. pdf\&typ=zusatztext.

Wankel, Charles, Matthew Marovich, und Jurate Stanaityte. 2010. Cutting-edge social media approaches to business education. Teaching with LinkedIn, Facebook, Twitter, Second Life, and blogs. Research in management education and development. Charlotte, NC: Information Age Publ.

Wolf, Karsten D., und Klaus Rummler. 2011. «Mobile Learning with Videos in Online Communities: The example of draufhaber.tv». Herausgegeben von Ben Bachmair, Norbert Pachler, und John Cook. MedienPädagogik: Zeitschrift für Theorie und Praxis der Medienbildung 19 (Mobile Learning in Widening Contexts: Concepts and Cases). https://doi.org/10.21240/ mpaed/19/2011.05.04.X. 